\title{
One loop matching factors for improved staggered four-fermion operators with improved glue
}

\section{Jongjeong Kim*, Weonjong Lee}

Lattice Gauge Theory Research Center, FPRD, and CTP

Department of Physics and Astronomy, Seoul National University, Seoul, 151-747, South Korea

E-mail: rvanguardephya.snu.ac.kr, wleedsnu.ac.kr

\section{Stephen R. Sharpe}

Physics Department, University of Washington, Seattle, WA 98195-1560

E-mail: sharpe@phys.washington.edu

\begin{abstract}
We present results for matching factors for staggered four-fermion operators constructed using HYP-smeared fat links both in the action and the operators. We use perturbation theory to calculate the matching factors and work to one-loop order. The new feaure of this work is the use of the Symanzik-improved gauge action, as opposed to the Wilson gauge action. Our results are needed for our ongoing calculation of weak matrix elements using HYP-smeared staggered valence quarks and operators on MILC lattices. We give explicit results for matching factors of the operator needed to calculate $B_{K}$. We compare the impact of the improvement of the gauge action on one-loop coefficients with that of mean-field improvement of the operators.
\end{abstract}

The XXVIII International Symposium on Lattice Field Theory, Lattice2010

June 14-19, 2010

Villasimius, Italy

\footnotetext{
*Speaker.
} 


\section{Introduction}

Matrix elements of electroweak four-fermion operators between hadronic states play a central role in constraining the parameters of the CKM matrix. The Wilson coefficients of the operators are typically calculated in a continuum renormalization scheme (usually the NDR [Naive Dimensional Regularization] scheme with $\overline{\mathrm{MS}}$ subtraction), so one needs to convert the matrix elements calculated on the lattice into this continuum scheme. The multiplicative factors that are needed (which are, in general, matrices) are called matching factors or "Z-factors". We consider here the matching factors for four-fermion operators constructed using HYP-smeared staggered fermions [1], and calculate them at one-loop order in perturbation theory. These are needed for our ongoing calculation of $B_{K}$ using HYP-smeared staggered fermions [2] on the MILC "asqtad" lattices [3].

The new feature compared to the previous calculation [4] of four-fermion matching factors is the use of the Symanzik-improved gluon action instead of the Wilson plaquette action. This extension is necessary because the MILC lattices are generated using the former action.

\section{Action and Feynman Rules}

For valence quarks we use the HYP-smeared action, which is simply the unimproved staggered action

$$
S_{f}=\frac{1}{2} \sum_{n, \mu} \eta_{\mu}(n) \bar{\chi}(n)\left(V_{\mu}(n) \chi(n+\hat{\mu})-V_{\mu}^{\dagger}(n-\hat{\mu}) \chi(n-\hat{\mu})\right)+m \sum_{n} \bar{\chi}(n) \chi(n),
$$

(where $\eta_{\mu}(n)=(-1)^{n_{1}+\cdots+n_{\mu-1}}$, and we have set the lattice spacing $a$ to unity) except that the links, $V_{\mu}$, are HYP-smeared fat links. By contrast, in the unimproved action the links are replaced by the original, "thin", links, $U_{\mu}$. The relation between these two types of links is given in Ref. [1]. What we need is the relation in perturbation theory and in momentum space:

$$
\begin{aligned}
& V_{\mu}(n)=\exp \left(i g B_{\mu}(n)\right) \\
& B_{\mu}(n)=\int_{-\pi}^{\pi} \frac{d^{4} k}{(2 \pi)^{4}} \sum_{v} h_{\mu v}(k) A_{v}(k) e^{i k \cdot(n+\hat{\mu} / 2)}+\mathscr{O}\left(A^{2}\right) .
\end{aligned}
$$

Here $A_{v}$ are the momentum space gluon fields obtained from the unimproved links, and $h_{\mu v}(k)$ is a smearing kernel containing all relevant information about the HYP smearing. For the HYP links whose coefficients are chosen to remove $\mathscr{O}\left(a^{2}\right)$ taste symmetry breaking at tree level $\left(\alpha_{1}=0.875\right.$, $\alpha_{2}=4 / 7$ and $\alpha_{3}=0.25$ in the notation of Ref. [1]), $h_{\mu v}(k)$ is $[5,6,7]$

$$
h_{\mu v}(k)=\delta_{\mu v} D_{\mu}(k)+\left(1-\delta_{\mu v}\right) \bar{s}_{\mu} \bar{s}_{v} \tilde{G}_{v, \mu}(k),
$$

where

$$
\begin{aligned}
D_{\mu}(k) & =1-\sum_{v \neq \mu} \bar{s}_{v}^{2}+\sum_{\substack{v<\rho \\
v, \rho \neq \mu}} \bar{s}_{v}^{2} \bar{s}_{\rho}^{2}-\bar{s}_{v}^{2} \bar{s}_{\rho}^{2} \bar{s}_{\sigma}^{2} \\
\tilde{G}_{v, \mu}(k) & =1-\frac{\left(\bar{s}_{\rho}^{2}+\bar{s}_{\sigma}^{2}\right)}{2}+\frac{\bar{s}_{\rho}^{2} \bar{s}_{\sigma}^{2}}{3}
\end{aligned}
$$


Here, $\mu \neq v \neq \rho \neq \sigma$ and $\bar{s}_{\mu}=\sin \left(k_{\mu} / 2\right)$. We recover the unimproved action (in which the links are thin) by setting $h_{\mu v}(k)=\delta_{\mu \nu}$.

One would expect the $\mathscr{O}\left(A^{2}\right)$ term in Eq. (2.3) to be needed at one-loop order, since it can give rise to tadpole diagrams. It turns out, however, that its contribution vanishes due to the $S U(3)$ projection used in HYP-smearing, as discussed in Refs. [8, 6, 9].

We use the tree level Symanzik-improved gluon action [10,11], which can be written as

$$
S_{g}=\frac{2}{g_{0}^{2}}\left[\frac{5}{3} \sum_{\mathrm{pl}} \operatorname{Re} \operatorname{Tr}\left(1-U_{\mathrm{pl}}\right)-\frac{1}{12} \sum_{\mathrm{rt}} \operatorname{Re} \operatorname{Tr}\left(1-U_{\mathrm{rt}}\right)\right],
$$

where "pl" and "rt" represent plaquette and rectangle, respectively. In fact, the MILC collaboration use a (partially) one-loop improved Symanzik gluon action to generate their configurations [12], and not the tree-level form. However, the one-loop corrections in the gluon action do not impact the matching factors of fermionic operators until two-loop level, and thus it is sufficient to use the tree-level gluon action. Similarly, the effect of dynamical quarks does not enter until two-loop level, so the fact that the valence and sea quarks have different actions does not impact our one-loop calculation.

Feynman rules for the staggered action and four-fermion operators can be found in literature, and we do not repeat them here. Quark propagators and vertices can be found in Refs. [13, 14, 8]. The generalization to various improved staggered fermions such as asqtad and HYP-smeared staggered fermions can be found in Ref. [5, 4]. The improved gluon propagator can be found in Refs. [10, 6, 7].

\section{Four-fermion Operators}

We use the hypercube construction of Ref. [15] for our staggered four-fermion operators. These come in two kinds, which differ in the contractions of their color indices. First we have "one color-trace" operators, labeled with a subscript $I$, whose form is

$$
\begin{aligned}
{[S \times F]\left[S^{\prime} \times F^{\prime}\right]_{I}(y)=\frac{1}{4^{4}} \sum_{A, B, A^{\prime}, B^{\prime}} } & {\left[\bar{\chi}_{a}^{(1)}(2 y+A) \overline{\left(\gamma_{S} \otimes \xi_{F}\right)}\right.} \\
A B & \left.\chi_{b}^{(2)}(2 y+B)\right] \\
\times & {\left.\left[\bar{\chi}_{a^{\prime}}^{(3)}\left(2 y+A^{\prime}\right) \overline{\left(\gamma_{S^{\prime}} \otimes \xi_{F^{\prime}}\right.}\right)_{A^{\prime} B^{\prime}} \chi_{b^{\prime}}^{(4)}\left(2 y+B^{\prime}\right)\right] } \\
& \times \mathscr{V}^{a b^{\prime}}\left(2 y+A, 2 y+B^{\prime}\right) \mathscr{V}^{\prime} b\left(2 y+A^{\prime}, 2 y+B\right) .
\end{aligned}
$$

Here $y \in \mathbf{Z}^{4}$ is the coordinate of hypercubes, hypercube vectors $S$ and $S^{\prime}$ denote the spins of the component bilinears, while $F$ and $F^{\prime}$ denote the tastes. Indices $a, b, a^{\prime}$, and $b^{\prime}$ denote colors, while superscripts $(i)$ for $i=1,2,3,4$ label different flavors (not tastes). Different flavors are chosen to forbid penguin diagrams. Two fat Wilson lines $\mathscr{V}^{a b^{\prime}}\left(2 y+A, 2 y+B^{\prime}\right)$ and $\mathscr{V}^{\prime} b\left(2 y+A^{\prime}, 2 y+B\right)$ ensure the gauge invariance of the four-fermion operators. A fat Wilson line $\mathscr{V}^{a} b^{\prime}\left(2 y+A, 2 y+B^{\prime}\right)$, for example, is constructed by averaging over all the shortest paths connecting $2 y+A$ and $2 y+B^{\prime}$, with each path formed by products of HYP-smeared links $V_{\mu}$. When we use the unimproved staggered action the Wilson lines are composed of unsmeared links, $U_{\mu}$. 
The second kind of operator is called "two color-trace", and takes the form

$$
\begin{aligned}
{[S \times F]\left[S^{\prime} \times F^{\prime}\right]_{I I}(y)=\frac{1}{4^{4}} \sum_{A, B, A^{\prime}, B^{\prime}} } & {\left[\bar{\chi}_{a}^{(1)}(2 y+A) \overline{\left(\gamma_{S} \otimes \xi_{F}\right)}{ }_{A B} \chi_{b}^{(2)}(2 y+B)\right] } \\
& \left.\times\left[\bar{\chi}_{a^{\prime}}^{(3)}\left(2 y+A^{\prime}\right) \overline{\left(\gamma_{S^{\prime}} \otimes \xi_{F^{\prime}}\right.}\right)_{A^{\prime} B^{\prime}} \chi_{b^{\prime}}^{(4)}\left(2 y+B^{\prime}\right)\right] \\
& \times \mathscr{V}^{a b}(2 y+A, 2 y+B) \mathscr{V}^{a^{\prime} b^{\prime}}\left(2 y+A^{\prime}, 2 y+B^{\prime}\right),
\end{aligned}
$$

where the subscript II denotes two color-traces. These operators differs from those with one colortrace by the choice of fat Wilson lines-here they connect within each bilinear, whereas for the one color-trace operators they connect between bilinears.

Following Refs. [8, 14, 16], we also consider mean-field improved operators. For the unimproved action and operators this is usually referred to as tadpole improvement [17]. For the HYPsmeared action and operators, mean-field improvement is achieved by rescaling the staggered fields and the link matrices:

$$
\chi \rightarrow \psi=\sqrt{v_{0}} \chi, \quad \bar{\chi} \rightarrow \bar{\psi}=\sqrt{v_{0}} \bar{\chi}, \quad V_{\mu} \rightarrow \tilde{V}_{\mu}=V_{\mu} / v_{0},
$$

where $v_{0}$ is the mean-field improvement factor defined by

$$
v_{0} \equiv\left[\frac{1}{3} \operatorname{Re} \operatorname{Tr}\left\langle V_{\mathrm{pl}}\right\rangle\right]^{1 / 4},
$$

with $V_{\mathrm{pl}}$ the plaquette composed of HYP-smeared links.

\section{Renormalization of Four-fermion operators}

Analytic formulae for one-loop perturbative corrections to the four-fermion operators for HYP-smeared staggered fermions with the Wilson plaquette action are given in Ref. [4]. It turns out that the generalization to using the improved gluon action rather than the Wilson plaquette action can be achieved simply by replacing the "composite" gluon propagator;

$$
\left(1 / \hat{k}^{2}\right) \sum_{\lambda} h_{\mu \lambda} h_{v \lambda} \rightarrow \sum_{\alpha \beta} h_{\mu \alpha} h_{v \beta} \mathscr{D}_{\alpha \beta}
$$

where $\mathscr{D}_{\alpha \beta}$ is the improved gluon propagator, while the Wilson gluon propagator is $\left(\delta_{\alpha \beta} / \hat{k}^{2}\right)$ with $\hat{k}^{2}=4 \sum_{\alpha} \sin ^{2}\left(k_{\alpha} / 2\right)$. This simplification holds both for bilinear operators [6] and four-fermion operators.

The Feynman diagrams which contribute to one-loop matching factors for four-fermion operators can be found in Ref. [4]. We have undertaken two independent calculations, using significantly different methods, to cross-check our results. Another check is obtained by using Fierz transformations, which interchange one and two color-trace operators. The form of these transformations relevant to our set-up is

$$
{\overline{\left(\gamma_{S} \otimes \xi_{F}\right)}}_{A B}{\overline{\left(\gamma_{S^{\prime}} \otimes \xi_{F^{\prime}}\right)_{A^{\prime} B^{\prime}}}}=\frac{1}{16} \sum_{M N}{\overline{\left(\gamma_{S} \gamma_{M}^{\dagger} \otimes \xi_{N}^{\dagger} \xi_{F^{\prime}}\right)}}_{A B^{\prime}}{\overline{\left(\gamma_{S^{\prime}} \gamma_{M} \otimes \xi_{N} \xi_{F}\right)}}_{A^{\prime} B},
$$

where $M, N, A, A^{\prime}, B$, and $B^{\prime}$ are hypercube vectors. 
The result of the calculation can be expressed as

$$
\vec{O}_{i}^{\mathrm{Cont},(1)}(\mu)=\sum_{j} Z_{i j}(\mu, a) \vec{O}_{j}^{\mathrm{Lat}}(1 / a)
$$

where the superscripts "Cont" and "Lat" represent the continuum and lattice respectively. The subindices $i$ and $j$ run over all combinations of spins and tastes of the four-fermion operators that are allowed by the lattice symmetries. The vectors on the four-fermion operators indicate that one and two color-trace operators, for given spins and tastes, are collected into a two-dimensional vector. We call the vector space in which these vectors live the "color-trace" space. The renormalization scale in the continuum scheme is $\mu$, and $a$ (now reintroduced) is the lattice spacing. Finally, the superscript "(1)" indicates that the continuum operator has been matched at one-loop order.

The matching factor $Z_{i j}$ takes the form

$$
Z_{i j}=\delta_{i j}+\frac{g^{2}}{(4 \pi)^{2}}\left[-\hat{\gamma}_{i j} \log (\mu a)+\hat{c}_{i j}\right] .
$$

where $\hat{\gamma}_{i j}$ and $\hat{c}_{i j}$ are, respectively, the anomalous dimension matrix and the finite coefficients. Apart from their explicit indices, both are also matrices in the color-trace space, as denoted by the "hats". The finite coefficients are given by the difference of finite terms in the continuum and lattice one-loop calculations,

$$
\hat{c}_{i j}=\hat{c}_{i j}^{\mathrm{Cont}}-\hat{c}_{i j}^{\mathrm{Lat}} .
$$

\section{Matching Factors for $B_{K}$}

We have obtained the matching factors for all four-fermion operators of the forms given above, i.e. for all choices of $S, S^{\prime}, F$ and $F^{\prime}$. Detailed results will be presented elsewhere [18]. We consider here only the matching factors needed for $B_{K}$. The continuum four-fermion operator relevant to $B_{K}$ is

$$
\mathscr{O}_{B_{K}}^{C o n t}=\left[\bar{s}^{a} \gamma_{\mu}\left(1-\gamma_{5}\right) d^{a}\right]\left[\bar{s}^{b} \gamma_{\mu}\left(1-\gamma_{5}\right) d^{b}\right]
$$

The corresponding operator on the lattice, in the "two spin-trace" formulation [19, 20], can be written at tree-level as

$$
\mathscr{O}_{B_{K}}^{\mathrm{Lat}} \equiv \sum_{i=1}^{4} \mathscr{O}_{i}^{\mathrm{Lat}}=\mathscr{V}_{I}+\mathscr{V}_{I I}+\mathscr{A}_{I}+\mathscr{A}_{I I}
$$

where

$$
\begin{array}{cl}
\mathscr{O}_{1}^{\mathrm{Lat}} \equiv \mathscr{V}_{I}=\left[V_{\mu} \times P\right]\left[V_{\mu} \times P\right]_{I}, & \mathscr{O}_{2}^{\mathrm{Lat}} \equiv \mathscr{V}_{I I}=\left[V_{\mu} \times P\right]\left[V_{\mu} \times P\right]_{I I}, \\
\mathscr{O}_{3}^{\mathrm{Lat}} \equiv \mathscr{A}_{I}=\left[A_{\mu} \times P\right]\left[A_{\mu} \times P\right]_{I}, & \mathscr{O}_{4}^{\mathrm{Lat}} \equiv \mathscr{A}_{I I}=\left[A_{\mu} \times P\right]\left[A_{\mu} \times P\right]_{I I}
\end{array}
$$

Here, $V_{\mu}, A_{\mu}$, and $P$ stand for the vector, axial-vector, and pseudo-scalar respectively. The taste of all the operators are taken to be the pseudo-scalar $P$, i.e. $F=\xi_{5}$.

At one-loop order, many lattice operators contribute to the matching formula. In practice, however, in our numerical calculation of $B_{K}$ we keep only those operators in which the bilinears 
have taste $\xi_{5} .{ }^{1}$ It turns out that only the four operators listed above have this taste. Thus what is relevant for our numerical calculation is the truncated one-loop matching formula

$$
\mathscr{O}_{B_{K}}^{\text {Cont, (1), Trunc }}=\sum_{i=1}^{4} c_{i} \mathscr{O}_{i}^{\mathrm{Lat},(1)}, \quad c_{i} \equiv\left[1+\frac{g^{2}}{(4 \pi)^{2}}\left(-4 \log (\mu a)+\left[-\frac{11}{3}-c_{i}^{\mathrm{Lat}}\right]\right)\right] .
$$

We use here the NDR scheme in the continuum, for which the finite coefficient is $-\frac{11}{3}$ for all four operators.

Results for $c_{i}^{\mathrm{Lat}}$ are given in table 1 for various choices of lattice operator. We see that, in most cases, the coefficients are slightly reduced by improving the gauge action, although the reduction is smaller than that obtained by for HYP fermions by mean-field improvement. As can be seen from Eq. (5.5), however, reducing the $c_{i}^{\text {Lat }}$ does not necessarily lead to matching factors closer to unity. A better measure is the range of the matching coefficients, i.e. the largest difference between them, for the continuum and logarithmic contributions then cancel. This quantity is also given in the Table, and one sees that it is moderately reduced by improving the gauge action.

\begin{tabular}{lrrrrrr}
\hline \hline & $(a)$ & $(b)$ & $(c)$ & $(d)$ & $(e)$ & $(f)$ \\
\hline Gluon action & Wilson & Sym & Wilson & Sym & Wilson & Sym \\
Quark action & Naive & Naive & HYP & HYP & HYP & HYP \\
Mean-field impr.? & $\mathrm{Y}$ & $\mathrm{Y}$ & $\mathrm{N}$ & $\mathrm{N}$ & $\mathrm{Y}$ & $\mathrm{Y}$ \\
\hline$c_{1}^{\text {Lat }}$ & -2.35 & -2.49 & -4.98 & -3.65 & -2.17 & -1.72 \\
$c_{2}^{\text {Lat }}$ & -12.92 & -11.53 & -11.17 & -8.58 & -5.49 & -4.73 \\
$c_{3}^{\text {Lat }}$ & -2.95 & -3.08 & -5.50 & -4.12 & -2.69 & -2.19 \\
$c_{4}^{\text {Lat }}$ & -3.73 & -2.90 & 1.01 & 1.09 & 1.01 & 1.09 \\
\hline Range & 10.57 & 9.04 & 12.18 & 9.67 & 6.50 & 5.82 \\
\hline \hline
\end{tabular}

Table 1: Results for $c_{i}^{\text {Lat }}$ for various choices of gauge and fermion action. We quote only two decimal places for brevity.

To give a sense of the size of the matching coefficients themselves, we show in table 2 results for the ultrafine MILC lattices ( $a \approx 0.045 \mathrm{fm}$ ), setting $\mu=1 / a$ ("horizontal matching"), and $g^{2} /(4 \pi)=0.2096$ (the value in the $\overline{M S}$ scheme at $\mu=1 / a$ ). For the actions we use in practice [column (d)] the one-loop corrections range between $\pm 8 \%$.

\begin{tabular}{rrrrrrr}
\hline \hline & $(a)$ & $(b)$ & $(c)$ & $(d)$ & $(e)$ & $(f)$ \\
\hline$c_{1}$ & 0.978 & 0.980 & 1.022 & 1.000 & 0.975 & 0.968 \\
$c_{2}$ & 1.154 & 1.131 & 1.125 & 1.082 & 1.030 & 1.018 \\
$c_{3}$ & 0.988 & 0.990 & 1.031 & 1.008 & 0.984 & 0.975 \\
$c_{4}$ & 1.001 & 0.987 & 0.922 & 0.921 & 0.922 & 0.921 \\
\hline \hline
\end{tabular}

Table 2: Values of $c_{i}$ for the MILC ultrafine lattice. Notation for actions as in table 1.

\footnotetext{
${ }^{1}$ The rationale for this is that we use external kaons with taste $\xi_{5}$. As shown in Ref. [21], however, leaving out the operators with other tastes leads to an error of $\mathscr{O}\left(\alpha_{s} m_{K}^{2} / \Lambda_{\chi}^{2}\right)$, which is of next-to-leading order in staggered chiral perturbation theory. This error must be accounted for when fitting.
} 


\section{Conclusion}

We have calculated the matching factors for four-fermion operators using various fermion and gauge actions. Most useful are our results for the fermion action and operators containing HYP-smeared links, and with the gluon action being Symanzik improved, for these are needed for our parallel calculation of $B_{K}$, and have been used in Ref. [2]. For these choices, the oneloop corrections are of moderate size, with the range of corrections being $\approx 10 \times \alpha_{s} /(4 \pi) \approx \alpha_{s}$, which is the naively expected size. The impact of improving the gauge action turns out to be numerically small, much less than the reduction in the size of the corrections caused by using mean-field improved operators.

\section{Acknowledgments}

The research of W. Lee is supported by the Creative Research Initiatives program (334820090015) of the NRF grant funded by the Korean government (MEST). The work of S. Sharpe is supported in part by the US DOE grant no. DE-FG02-96ER40956.

\section{References}

[1] A. Hasenfratz and F. Knechtli, Phys. Rev. D 64, 034504 (2001) [arXiv:hep-lat/0103029].

[2] T. Bae, Y. -C. Jang, C. Jung et al., [arXiv:1008.5179 [hep-lat]].

[3] C. Bernard et al., Phys. Rev. D 64, 054506 (2001) [arXiv:hep-lat/0104002].

[4] W. Lee and S. R. Sharpe, Phys. Rev. D 68, 054510 (2003) [arXiv:hep-lat/0306016].

[5] W. Lee and S. R. Sharpe, Phys. Rev. D 66, 114501 (2002) [arXiv:hep-lat/0208018].

[6] J. Kim, W. Lee and S. R. Sharpe, PoS LAT2009, 201 (2009) [arXiv:0910.5586 [hep-lat]].

[7] J. Kim, W. Lee and S. R. Sharpe, Phys. Rev. D 81, 114503 (2010) [arXiv:1004.4039 [hep-lat]].

[8] A. Patel, S. R. Sharpe, Nucl. Phys. B395, 701-732 (1993). [hep-lat/9210039].

[9] W. Lee, Phys. Rev. D 66, 114504 (2002) [arXiv:hep-lat/0208032].

[10] P. Weisz, Nucl. Phys. B 212, 1 (1983).

[11] M. Luscher and P. Weisz, Commun. Math. Phys. 97, 59 (1985) [Erratum-ibid. 98, 433 (1985)].

[12] M. G. Alford, W. Dimm, G. P. Lepage et al., Phys. Lett. B361, $87-94$ (1995). [hep-lat/9507010].

[13] D. Daniel and S. Sheard, Nucl. Phys. B 302, 471 (1988).

[14] N. Ishizuka and Y. Shizawa, Phys. Rev. D 49, 3519 (1994) [arXiv:hep-lat/9308008].

[15] H. Kluberg-Stern et al., Nucl. Phys. B 220, 447 (1983).

[16] W. Lee, Phys. Rev. D64, 054505 (2001). [hep-lat/0106005].

[17] G. P. Lepage, P. B. Mackenzie, Phys. Rev. D48, 2250-2264 (1993). [hep-lat/9209022].

[18] J. Kim, W. Lee and S. R. Sharpe, in preparation.

[19] S. R. Sharpe, A. Patel, R. Gupta et al., Nucl. Phys. B286, 253 (1987).

[20] G. Kilcup, R. Gupta and S. R. Sharpe, Phys. Rev. D 57, 1654 (1998) [arXiv:hep-lat/9707006].

[21] R. S. Van de Water and S. R. Sharpe, Phys. Rev. D 73, 014003 (2006) [arXiv:hep-lat/0507012]. 